LA WRENCE LWWEAMCAE NATIONAL LABDPATOAY

\title{
Simulating Electron Cloud Effects in Heavy-Ion Beams
}

R.H. Cohen, A. Friedman, S.M. Lund, A.W. Molvik, T. Azevedo, J.-L. Vay, P. Stoltz and S.Veitzer

June 2004

HIF Symposium, Princeton, N.J., 6/7/04 
This document was prepared as an account of work sponsored by an agency of the United States Government. Neither the United States Government nor the University of California nor any of their employees, makes any warranty, express or implied, or assumes any legal liability or responsibility for the accuracy, completeness, or usefulness of any information, apparatus, product, or process disclosed, or represents that its use would not infringe privately owned rights. Reference herein to any specific commercial product, process, or service by trade name, trademark, manufacturer, or otherwise, does not necessarily constitute or imply its endorsement, recommendation, or favoring by the United States Government or the University of California. The views and opinions of authors expressed herein do not necessarily state or reflect those of the United States Government or the University of California, and shall not be used for advertising or product endorsement purposes. 


\title{
Simulating Electron Cloud Effects in Heavy-Ion Beams
}

\author{
R. H. Cohen, ${ }^{1}{ }^{*}$ A. Friedman, ${ }^{1}$ S. M. Lund,${ }^{1}$ A. W. Molvik, ${ }^{1}$ T. Azevedo, ${ }^{2}$ J.-L. Vay, ${ }^{2}$ P. Stoltz,${ }^{3}$ and S. Veitzer ${ }^{3}$ \\ ${ }^{1}$ Lawrence Livermore National Laboratory, P.O. Box 808, Livermore CA 94550 \\ ${ }^{2}$ Lawrence Berkeley National Laboratory, Berkeley, CA 94720 \\ ${ }^{3}$ TechX Corp., Boulder CO \\ (Dated: June 4, 2004 DRAFT)
}

\begin{abstract}
Stray electrons can be introduced in heavy ion fusion accelerators as a result of ionization of ambient gas or gas released from walls due to halo-ion impact, or as a result of secondary- electron emission. We summarize here results from several studies of electron-cloud accumulation and effects: (1) Calculation of the electron cloud produced by electron desorption from computed beam-ion loss; the importance of ion scattering is shown; (2) Simulation of the effect of specified electron cloud distributions on ion beam dynamics. We find electron cloud variations that are resonant with the breathing mode of the beam have the biggest impact on the beam (larger than other resonant and random variations), and that the ion beam is surprisingly robust, with an electron density several percent of the beam density required to produce significant beam degradation in a 200-quadrupole system. We identify a moderately growing instability associated with resonance with the breathing mode. (3) Preliminary investigations of a long-timestep algorithm for electron dynamics in arbitrary magnetic fields.

PACS numbers:

Keywords:
\end{abstract}

\section{INTRODUCTION}

Heavy-Ion-Fusion (HIF) accelerators, like other positive-charge-particle accelerators, are subject to contamination by stray electrons, which can be electrostatically trapped by the ion beam potential. The phenomenon has been documented in a range of positivecharge-particle accelerators dating back to the 1960's [1], and has been the subject of dedicated international meetings; see Refs. [2] and [3] and references therein.

Common to all applications is that the electron cloud is an uncontrolled source of negative charge that can produce alteration of the ion beam dynamics, possibly leading to beam deflection, increased beam emittance, envelope size, and halo, and also potentially driving electronion instabilities. On the other hand induction HIF accelerators have a number of distinguishing features that impact the nature and modeling of electron clouds, such as their relatively long pulse length, the economics-driven large pipe fill factor, the relatively large portion of the accelerator occupied by magnets, the localization of the beam pipe to within magnets, and the presence of electrostatic accelerating fields in between magnets. Because of these features, the dominant source of electrons (at sufficiently good vacuum) is desorption of gas and electrons from beam ions impacting walls, with the former dominating for long (multi- $\mu$ s pulses).

In this paper we summarize results from studies of electron cloud buildup and effects, in particular a chain of calculations to determine the electron cloud produced by electron desorption, and simulations of ion beams in

\footnotetext{
${ }^{*}$ rcohen@llnl.gov
}

the presence of various prescribed electron-cloud distributions. The work will be described in more detail in a longer publication [4], which also includes a discussion of the electron dynamics in HIF accelerators and a map of our plan for self-consistent electron and ion simulation.

\section{ELECTRON CLOUD ACCUMULATION SIMULATIONS}

We show here sample calculations of the electron cloud resulting from desorption of electrons when primary and secondary (scattered) ions strike the walls. The simulation was done for a $2 \mathrm{MeV}$ Potassium beam in a 200quadrupole (100 lattice-period) transport system, with parameters similar to the magnetic quadrupoles in the High Current Experiment (HCX) at Lawrence Berkeley National Laboratory. Each magnet is identical, and represented by gridded field data from a 3D magnet calculation. The magnets are $10.2 \mathrm{~cm}$. long, have a radial field gradient of $90.51 T / m$. The gaps alternate in length between $6.1 \mathrm{~cm}$ and $18.5 \mathrm{~cm}$ ("syncopated lattice"), giving a total lattice period of $45 \mathrm{~cm}$. The beam is taken to be bounded by a circular-cross-section beam pipe, of radius $2.95 \mathrm{~cm}$.

This simulation was done through a sequence of calculations: using the WARP particle simulation code [5], a two-dimensional slice of the ion beam was followed through the lattice (with a small misalignment of magnets to exaggerate beam halo scrape-off. From the ensemble of scraped-off ions, a population of scattered ions was calculated from the TRIM surface Monte Carlo code [6], and these were then followed in WARP until their next wall impact. For both the primary and secondary ion impacts, the number and velocity distribution of elec- 


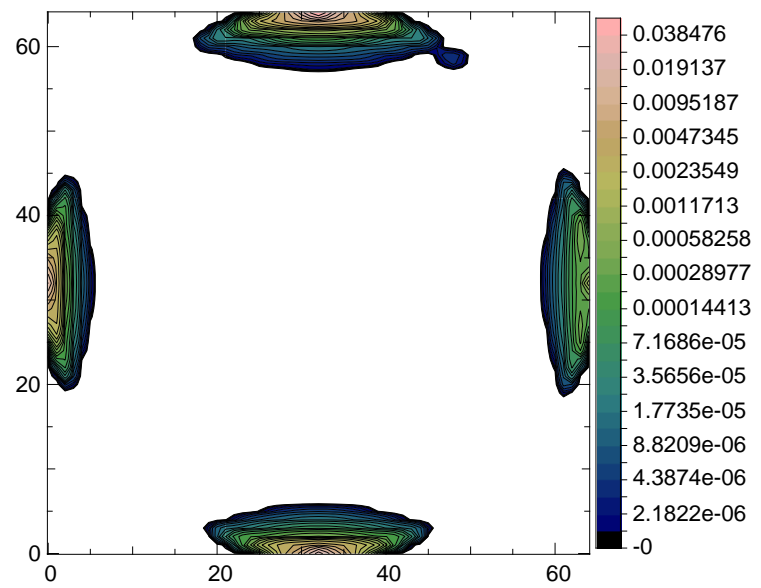

FIG. 1: $x-y$ projection of electron cloud density retaining only electrons desorbed by primary beam-ion impact at walls

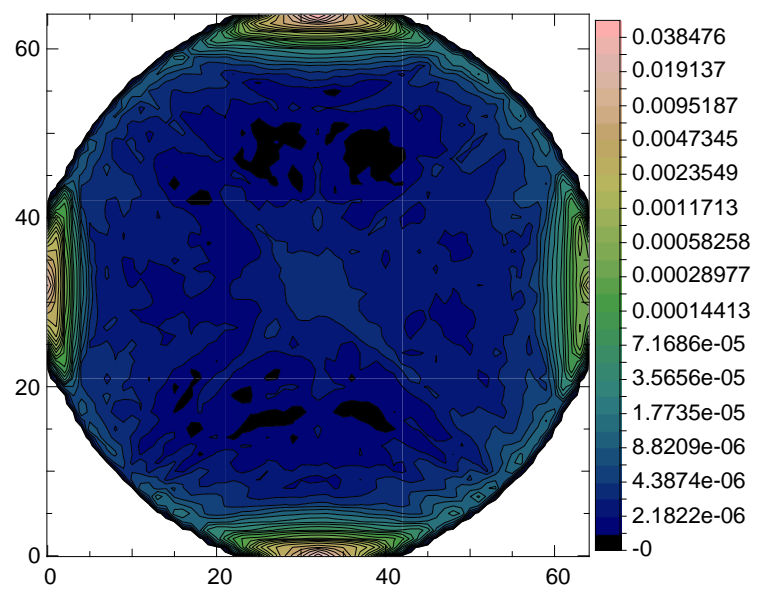

FIG. 2: $x-y$ projection of electron cloud density retaining electrons desorbed by impact of primary beam and scattered ions at walls

trons desorbed was calculated using a fit to the experimental data from Ref. [7]. The resulting electron population is followed in WARP (3D) for 4000 (sub-cyclotronperiod) timesteps, enough for several electron bounces. Electrons that are reach the wall are removed from the calculation. The time-integrated electron charge density is formed by incrementally depositing the weighted electron charge density onto the grid.

The three-dimensional results for the electron density, projected on two a two-dimensional plane transverse to the beam, are shown in Fig. 1 keeping only the electrons from the primary (beam) ion loss, and in Fig. 2 keeping the electrons desorbed from loss of both the primary and scattered ions. We see that, without the scattered ions, virtually all of the electron density is located at the edge near the $0,90,180$, and 270 degree points. This is because all of the scrape-off of ions occurs near the tips of the elliptical beam envelope distortion midway through each quadrupole magnet; the resulting electrons are con- fined on short field lines which stay close to the wall. Some of the scattered ions, on the other hand, hit the wall where there are field lines that reach deep into the interior of the beam. Hence, in Fig. 2, we see that, while the electron density is still pleaked near the wall, there is now some electron density everywhere.

We have recalculated the electron density using a new long-timestep electron mover, which interpolates between a full-electron-dynamics push [8] and drift kinetics. This interpolated mover preserves physically correct parallel and drift motion and gyration radius. The results are almost identical. The mover will be described in more detail in a separate publication.

The small local maximum near the center of the pipe is a recurring feature in simulations we have done. We tentatively attribute it to nonadiabatic scattering [9], which preferentially affects electrons which pass close to the center and can transiently trap electrons in the magnetic well.

\section{EFFECT OF MODEL ELECTRON CLOUDS ON ION DYNAMICS}

We summarize here an extensive set of studies, presented in full in Ref. [4], of the effects of prescribed model electron clouds on ion beam dynamics. The model consists of a specified, frozen-in-time negative charge distribution. The choice of models is motivated by consideration of long-pulse machines (in particular, current experiments or the injector end of a driver) where desorbed neutral gas is likely to be the dominant source and expected to produce an electron cloud which is roughly localized to the ion beam envelope and (because of magnetic confinement) within the quadrupoles - but with an assortment of possible variations, such as we explore.

Our base model electron distribution is constant within the (no-electron-cloud) beam envelope and within a quadrupole, and the same constant within each quadrupole. We have run ion beam slice simulations through the same 200-quadrupole system discussed in the preceding section, with this base model, and with a number of variations added to this distribution: (i) electron density varying from quadrupole to quadrupole; (ii) centroid of the electron cloud displaced by an amount which is constant within a quadrupole but varies from quadrupole to quadrupole; (iii) addition of a radially parabolically varying (zero-volume-integrated) density contribution; this perturbation allows the electron cloud to be peaked or hollow; and (iv) addition of an additional stretch along one axis and shrink along the other of the electron cloud envelope, by a factor that varyie from quad to quad. We consider axial variations which are either random from quadrupole to quadrupole; or sinusoidally varying with the quadrupole number. In the case of the centroid variation, for the random cases the direction of displacement also be random; for sinusoidal cases, the centroid rotates (with fixed fractional 

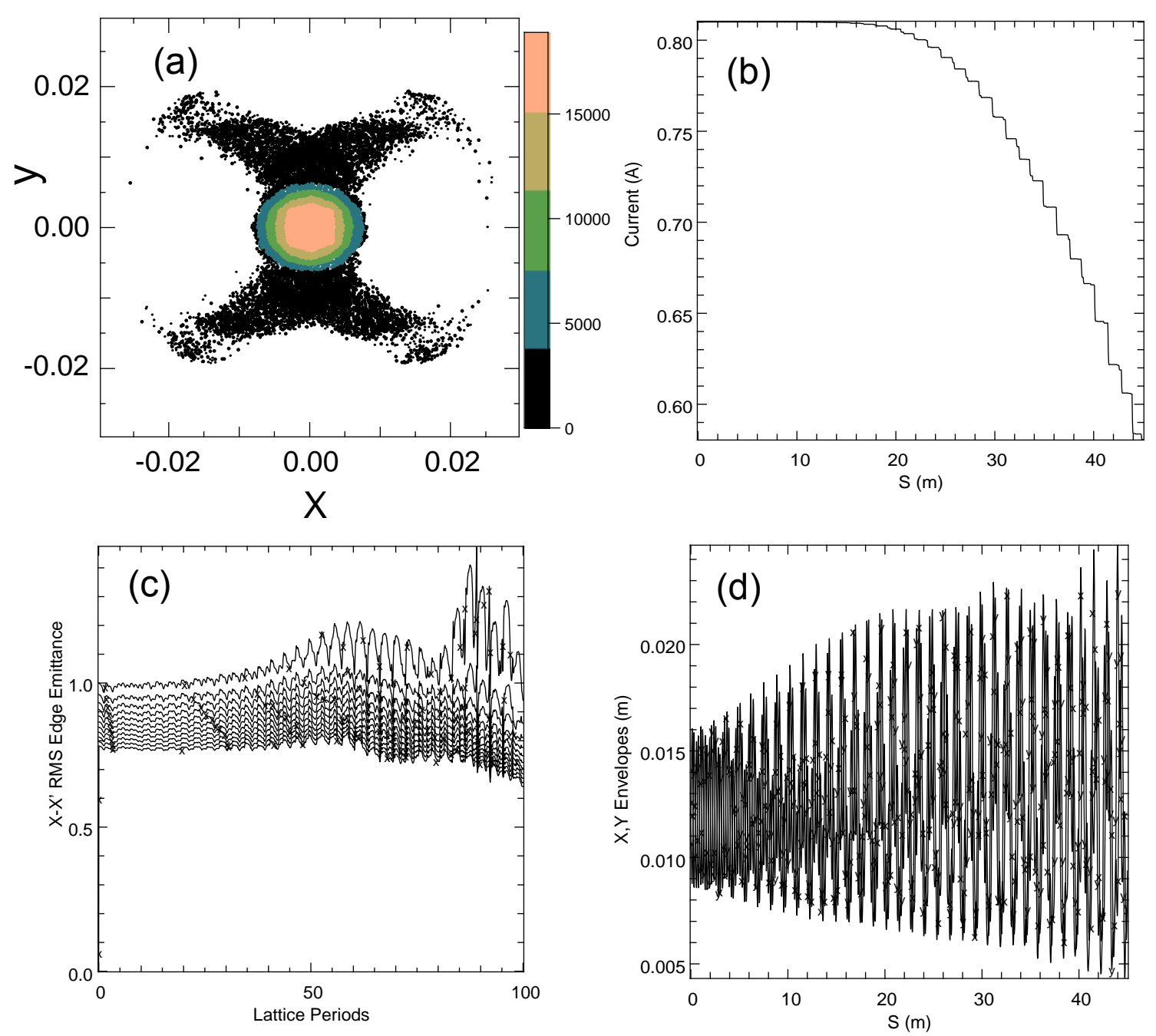

FIG. 3: Results for resonant, sinusoidally varying, $100 \%$ modulated electron density, with mean $n_{e} / n_{b}=0.05$ : (a) $x-y$ scatter plot of ions near end of magnet array; (b) beam current versus axial position; (c) emittance in $x$ plane versus axial position for various percentages of the beam current enclosed by nested ellipses in phase space (90\% to $100 \%$ in $1 \%$ increments); (d) $x$ and $y$ envelope semi-axes (the RMS extents times two) versus axial position

displacement relative to the envelope) as one progresses from quadrupole to quadrupole. In all cases we consider the extreme limit of $100 \%$ modulation, displacement, etc, from the base case. The random variations are representative of what might be expected from random alignment errors and random changes in wall conditions. The sinusoidal variations allow us to explore electrons from, and possible resonance with, breathing, centroid oscillation, and quadrupole oscillation modes of the beam. For resonant perturbations, we scan in perturbation wavelength about the nominal values derived from envelope equations [10, 11], and report results for at the wavelength of maximum beam current loss. The appropriate resonances are the breathing mode for the amplitude and shape variations, beam centroid oscillations for cloud centroid variations, and the quadrupole mode for the ellipticity modulation.

Our results indicate several trends. First, the base model (the same constant electron density $n_{e}$ in each quadrupole) has surprisingly little impact on the beam. Even for $n_{e} / n_{b}=0.2$, where $n_{b}$ is the beam density, there is surprisingly little effect on beam quality: imperceptable effects on plots of the emittance and beam envelope, negligible (less than 1 part in $10^{4}$ ) loss of beam current, and a discernable but small growth in the halo. Second, within each category (resonant, sinusoidal), density variations are the most effective in producing envelope growth and current loss, followed by shape variations, centroid oscillations, and ellipticity modulation (the last one studied only for the resonant case). On the other hand ellipticity modulation, which has the least effect on the beam current, is the only perturbation to have a signficant effect on emittance inside of the outermost few percent of beam particles. These effects are illustrated in Fig. 3, which shows an $x-y$ scatter plot, beam current, emittance of beam fractions, and envelope ver- 


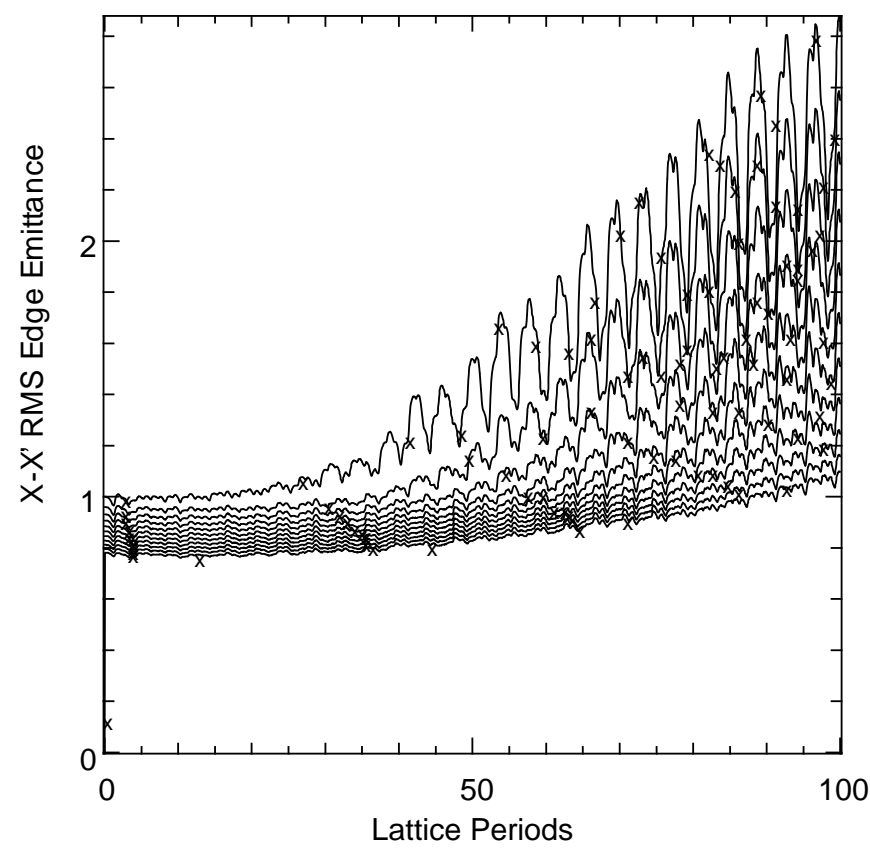

FIG. 4: Emittance versus beam fraction for resonant, sinusoidally varying electron cloud ellipticity, with $n_{e} / n_{b}=0.05$

sus axial position $z$ for the case of resonant sinusoidally varying electron density, and in Fig. 4, which shows the emittance for resonant elliticity variation. Third, the resonant perturbations are (not surprisingly) more effective in perturbing the ion beam than random perturbations. The largest resonant perturbation (density modulation) produces $28 \%$ beam current loss for a mean $5 \% n_{e} / n_{b}$, whereas a random density variation with mean $20 \% n_{e} / n_{b}$ produces only an $11 \%$ loss. Fourth, runs at peak current loss for several different resonant electron densities suggests a scaling of current loss with electron density slightly faster than $\left(n_{e} / n_{b}\right)^{2}$ for the (worst) case of resonant density modulation. Fifth, the resonant interactions are quite narrow in wavenumber. The full width at half maximum of the beam loss versus wavenumber is about $3 \%$ for the amplitude, radial shape, and centroid variations, and about twice that for the ellipticity variation.

Finally, an examination of plots of the electron density modulation and the resultant envelope excursions indicates that the two variations are approximately in phase until the beam envelope becomes comparable to the pipe radius and so produces significant scrape-off. This suggests an instability: where the envelope peaks, more electrons will be produced; these are relatively immobile in the beam direction because of confinement by the magnetic field. Hence the increment in density will persist, leading to further localized beam loss. Because of the stationarity of the electron perturbation, constant wavenumber is not required for the instability to exist; hence it can occur in an accelerating beam. A semiempirical estimate of the instantaneous growth rate can be constructed from linearized equations for the electron and neutral-density growth rates, with the system closed by a relationship between the beam scrape-off current and the resonant electron density, which we take (empirically) from our mini-scan of loss versus $n_{e}$ discussed above. We make a number of simplifying assumptions: a coasting beam, dominance of gas desorption, neglect of the time of flight of neutrals, neglect of any velocity tilt, equating the cross sectional area of the beam and beam pipe, and the approximation that $d I / d z \approx \Delta I / L$ where $I$ is beam current, $\Delta I$ its total loss and $L$ is the accelerator length.

The result is

$$
\gamma \sim\left(\frac{n_{b}}{n_{e}} \frac{2\left\langle\sigma_{i} v_{b}\right\rangle \kappa_{n} \Delta I}{q_{i} V}\right)^{1 / 2}
$$

where $\sigma_{i}$ is the ionization cross section, $v_{b}$ is the beam velocity, $\kappa_{n}$ is the number of desorbed neutrals per incident ion, and $V$ is the volume of the beam line (cross sectional area of beam pipe times length of the magnetic focussing section of the accelerator. Inserting results and parameters from Fig. 3, and taking $\left\langle\sigma_{i} v_{b}\right\rangle=1 \times 10^{-15}$ $\mathrm{cm}^{2}, \kappa_{n}=10^{4}$, we estimate a growth time $\gamma^{-1} \sim 1 \mu \mathrm{s}$. This is a moderate growth time, of the order of, but sufficiently smaller than, the pulse duration $(\sim 4 \mu \mathrm{s})$, that it motivates a more detailed calculation. We will report on such a study in the future.

\section{DISCUSSION}

We have presented simulations which exploit pieces of a developing capability [4? ] for heavy-ion-beam simulations which self-consistently include electrons. Specifically, these are models for electron desorption, ion reflection, and efficient dynamics for electrons and ions. The physics problems studied are electron accumulation resulting from ion-impact-produced electron desorption, effects of specified electron cloud perturbations on ion beam evolution, and a gas-desorption-induced instability.

The electron desorption studies indicate the importance of retaining ion scattering. The specified electroncloud studies indicate that ion beams are quite robust to constant electron cloud density, that a beam-wise variation in the electron density is more effective than the other variations considered in producing beam current loss while elliptical distortions are more effective at producing bulk emittance growth, and that resonant perturbations are more effective than random ones. These studies also lead to the inference of the gas-desorption instability and an estimate of its moderate growth rate.

One aspect of these studies is that they indicate that the impact of electron clouds is a rich and complex phenomenon, not simply characterized by the evolution of a few beam moments. For example, examination of the halo produced by several of the perturbation models indicates a halo that can be quite different in shape from the 
beam envelope; figure 3 provides an example. Once such a halo forms, electron desorption from primary beam loss can produce electrons on field lines which access the beam center, changing the picture from that described in Sec. 2. This example illustrates the need for a fully self-consistent simultaneous electron and ion simulation capability, such as we are developing.

\section{Acknowledgments}

We thank D. Grote for invaluable assistance with the WARP code. This work was performed under the aus- pices of the U.S. Department of Energy by Lawrence Livermore National Laboratory under contract W7405ENG-48 and by Lawrence Berkeley National Laboratory under contract DE-AC03-76F00098.
[1] G. Budker, G. Dimov, and V. Dudnikov, Sov. Atom. E. 22, 5 (1967)

[2] ECLOUD $02 \quad$ Workshop, CERN Report CERN-2002-001, http:// wwwslap.cern.ch/collective/ecloud02/proceedings/

[3] ECLOUD 04 Workshop proceedings, http://icfaecloud04.web.cern.ch/icfa-ecloud04/agenda.html

[4] R.H. Cohen, A. Friedman, S.M. Lund, A.W. Molvik, T. Azevedo, J.-L. Vay, P. Stoltz and S. Veitzer, to be submitted to PRSTAB (2004).

[5] D. P. Grote, A. Friedman, I. Haber, "ThreeDimensional Simulations of High Current Beams in Induction Accelerators with WARP3d," Fus. Eng. \& Des., 32-33, $193 \quad$ (1996) and http://hif.lbl.gov/theory/WARP_summary.html

[6] J. F. Ziegler, http://www.srim.org/

[7] A. W. Molvik, D. Baca, F.M. Bieniosek, R.H. Cohen, A.
Friedman, E.P. Lee, S.M. Lund, L. Prost, A. Sakumi, P.A. Seidl, J.L. Vay, "Initial Experimental Studies of Electron Accumulation in a Heavy-Ion Beam", Particle Accelerator Conference, Portland OR, 2003, paper TOPC004; http://epaper.kek.jp/p03/INDEX.HTM

[8] J. P. Boris, "relativistic Plasma Simulation - Optimization of a Hybrid Code," Proc. 4th International Conference on Numerical Simulation of Plasmas, Washington DC, Nov. 1970, p. 3.

[9] Howard, J.E., Phys. F̂luids 14, 2378 (1971).

[10] M. Reiser, "Theory and design of charged particle beams", John Wiley \& Sons, Inc., New-York (1994)

[11] J. J. Barnard and S. M. Lund, "Space charge effects in beam transport", course notes, U.S. Particle Accelerator School, Boulder, Colorado (2001), and private communication (2004) 